\title{
Smoking is ok as long as I eat healthily: Compensatory Health Beliefs and their role for intentions and smoking within the Health Action Process Approach
}

\author{
Theda Radtke ${ }^{\mathrm{a} *}$, Urte Scholz ${ }^{\mathrm{b}}$, Roger Keller ${ }^{\mathrm{a}}$ and Rainer Hornung ${ }^{\mathrm{a}}$ \\ ${ }^{a}$ Department of Psychology, Social and Health Psychology, University of Zurich, \\ Binzmühlestrasse, 14/Box 14, 8050 Zurich, Switzerland; ' Institute of Psychology, \\ Health Psychology, University of Bern, Alpeneggstr. 22, 3012 Bern, Switzerland
}

\begin{abstract}
Compensatory Health Beliefs (CHBs) are defined as beliefs that the negative consequences of unhealthy behaviours can be compensated for by engaging in healthy behaviours. CHBs have not yet been investigated within a framework of a behaviour change model, nor have they been investigated in detail regarding smoking. Thus, the aim of this study was to investigate on a theoretical basis whether smoking specific $\mathrm{CHBs}$, as a cognitive construct, add especially to the prediction of intention formation but also to changes in smoking behaviour over and above predictors specified by the Health Action Process Approach (HAPA). The sample comprised 385 adolescent smokers (mean age: 17.80). All HAPA specific variables and a smoking specific $\mathrm{CHB}$ scale were assessed twice, 4 months apart. Data were analysed using structural equation modelling. Smoking specific CHBs were significantly negatively related to the intention to stop smoking over and above HAPA specific predictors. Overall, 39\% of variance in the intention to quit smoking was explained. For the prediction of smoking, $\mathrm{CHBs}$ were not able to explain variance over and above planning and self efficacy. Thus, smoking specific CHBs seem mainly important in predicting intentions but not behaviour. Overall, the findings contribute to the understanding of the role of smoking specific CHBs within a health behaviour change model.
\end{abstract}

Keywords: Compensatory Health Beliefs; Health Action Process Approach; smoking; adolescents; intention

\section{Theoretical background}

Smoking is an important risk factor for many diseases, such as cancer (e.g. lung or stomach cancer), myocardial infarction and stroke (American Cancer Society, 2000). Nevertheless, smoking is still prevalent, especially among young people (Shafey, Eriksen, Ross, \& Mackay, 2009; WHO, 2007). In Switzerland, 27\% of the 15- to 19-year-olds are smokers (boys: $31 \%$, girls: $23 \%$; Keller, Radtke, Krebs, \& Hornung, 2011). As national and representative studies have shown, most adolescent

*Corresponding author. Email: theda.radtke@psychologie.uzh.ch 
smokers are quite knowledgeable about the harmful effects of smoking and also report a desire to quit smoking (53\% in Switzerland; Bak \& Piko, 2007; Keller et al., 2011; Pinel, Assanand, \& Lehmann, 2000; Radtke, Keller, Krebs, \& Hornung, 2010). Unfortunately, attempts at quitting have shown very low success rates (Keller et al., 2011; Mermelstein, 2003). Either barriers emerge or people give into temptation (Schwarzer \& Luszczynska, 2008).

When people are faced with the choice between giving into temptation (e.g. smoking a cigarette at a party) and the pursuit of their initial intention (e.g. to give up smoking), they experience a mental conflict. This mental conflict may be experienced for various reasons, such as (1) fear that the unhealthy behaviour will result in disease, (2) conflict with a valued self-perception (e.g. being somebody who lives a healthy life), or (3) a discrepancy in self-expectations (e.g. to stop smoking successfully; E. Harmon-Jones \& C. Harmon-Jones, 2007). The Compensatory Health Belief (CHB) model (Rabiau, Knäuper, \& Miquelon, 2006) proposes that $\mathrm{CHBs}$ act as a strategy to resolve this mental conflict.

CHBs are defined as beliefs that the negative consequences of unhealthy behaviours (e.g. smoking) can be compensated for or neutralised through the performance of healthy behaviours (e.g. eating fruit or exercising). CHBs are elicited by temptations or are generated after an indulgence in which a person fails to resist a temptation (Kronick \& Knäuper, 2010). It is further assumed that CHBs reduce cognitive dissonance by justifying unhealthy behaviour choices with the intention of engaging in other healthy behaviour. This does not guarantee, however, that people actually carry out the intended compensatory behaviour (e.g. exercise the next morning), because the initial dissonance may weaken over time, causing the need to compensate for the unhealthy behaviour to fade. Thus, people wanting to give up an unhealthy behaviour, like smoking, are impeded by CHBs because they can engage in unhealthy behaviour without feeling guilty about its negative health effects. Over time, a pathogenesis of diseases might be the consequence (Knäuper, Rabiau, Cohen, \& Patriciu, 2004; Rabiau et al., 2006).

So far there has been little research on CHBs. For this reason, there are few studies about $\mathrm{CHBs}$ in adolescents and few studies about smoking behaviour. However, those studies that exist provide evidence that CHBs are associated with poorly maintained self-care behaviour in adolescents (Rabiau, Knäuper, Nguyen, Sufrategui, \& Polychronakos, 2009). In addition, studies have shown that adolescent smokers activate smoking-specific $\mathrm{CHBs}$ to diminish the perceived health risk caused by smoking. The studies also demonstrated that smoking-specific CHBs are significantly, negatively related to the readiness to stop smoking (Radtke, Scholz, Keller, Knäuper, \& Hornung, 2011; Scheffel \& Schou, 2007). In line with this, Knäuper et al. (2004) have shown that substance-use specific CHBs are directly, positively related to smoking-/alcohol-related risk behaviour.

Nevertheless, CHBs have not been investigated within the framework of a behaviour change model until now. The CHB model (Rabiau et al., 2006) mainly explains the generation of CHBs, as well as the implementation of compensatory health behaviour, but it does not explain the relevance of CHBs for intention formation and behaviour changes. It only assumes that CHBs influence the process of thinking about different healthy and unhealthy behaviour choices in a way that they inhibit the intention for future health behaviour and thus impede the healthy behaviour. 
Therefore, it is necessary to test whether CHBs add to predictions of intention formation and behaviour change - over and above the constructs already included in a behaviour change model - in order to demonstrate the incremental validity of the new construct. Only by applying this test, results might be able to indicate whether or not the theory might be expanded by the new construct. Therefore, this study intended to integrate smoking-specific CHBs into an existing theoretical model of behaviour change. For this study, the Health Action Process Approach (HAPA; Schwarzer, 1992) constituted the theoretical background because it is a parsimonious, well-established model in health behaviour research (Schwarzer, 2008).

The Health Action Process Approach suggests that the adoption, initiation, and maintenance of health behaviours should be considered as a structured process (Schwarzer, 1992). Therefore, it differentiates between a pre-intentional motivational phase and a post-intentional volitional phase. Both main phases are defined by different social-cognitive processes that influence the way people become motivated and turn to action. The predictors of behavioural intention are risk perception, outcome expectancies, and self-efficacy. Risk perception, defined as a perceived vulnerability to one's own health, is understood as the distal starting point of a deliberation process for health behaviour change. Outcome expectancies are a consideration of the pros and cons of behaviour consequences and, next to self-efficacy, outcome expectancies are one of the major predictors of intention. Self-efficacy is defined as a feeling of competency regarding a person's ability to overcome barriers in everyday life or, in this case, in health behaviour change (Bandura, 1997). It is assumed that self-efficacy is relevant in both phases of the HAPA.

The formation of intention leads to the volition phase where the intention has to be translated into action. In this phase, self-regulatory processes, such as action plans, that mediate between the intention and the action are defined. Action plans are understood as the formation of concrete plans about when, where and how to implement intended behaviour (Sniehotta, Schwarzer, Scholz, \& Schüz, 2005).

Good empirical evidence exists for the HAPA model (Schwarzer et al., 2007). Furthermore, based on the first studies of the HAPA model concerning smoking behaviour (Scholz, Nagy, Göhner, Luszczynska, \& Kliegel, 2009; Schwarzer \& Luszczynska, 2008), it could be stated that the HAPA is applicable to smoking behaviour. Beyond this, it should be noted that the HAPA is based on the assumption that a person is a rational decision maker. The role of emotions and situational variables (e.g. cigarettes in a drawer) is rather neglected (Pitts \& Phillips, 1998). Thus, the integration of CHBs into the HAPA might be of special benefit to the model. As mentioned above, CHBs are activated as a consequence of motivational conflicts that arise from the interplay between affective states (desire, or anticipated pleasure, e.g. to smoke) and motivation (goals, e.g. quitting smoking). Therefore, it is reasonable to investigate CHBs by using the theoretical framework of the HAPA.

\section{Aims of the study}

Due to the concepts mentioned above, and especially because CHBs have not yet been investigated within the framework of a health behaviour change model, it was decided to investigate the CHBs within the framework of the HAPA. 
The CHB model (Rabiau et al., 2006) assumes that the CHBs are activated by a motivational conflict between the desire for a pleasant behaviour and the health goal. Individuals deliberate about whether to engage in the healthy, but unpleasurable behaviour or in the alternative behaviour with its unhealthy, but more pleasurable outcomes. This may influence the intention for the health behaviour. The activation of CHBs results in a reduction of the motivational conflict and may also lower the strength of the intention to change the unhealthy behaviour. This is because individuals believe that there is no need to give up the behaviour, as they can neutralise its negative effects by a compensatory behaviour. Therefore, the added value of smoking-specific CHBs was especially expected for the process of intention formation within the motivational phase of the HAPA. This theoretical assumption could also be confirmed by a recent study demonstrating that smoking-specific $\mathrm{CHBs}$ are significantly, negatively related to the readiness to stop smoking (Radtke et al., 2011). Moreover, this is in line with research about strategies for reducing cognitive dissonance, which has shown that dissonance-reducing strategies are negatively related to intentions to quit in smokers (Lee et al., 2009; Oakes, Chapman, Borland, Balmford, \& Trotter, 2004). Consequently, the main aim of this study was to examine if smoking-specific CHBs are able to add to the prediction of the intention to quit smoking over and above the standard HAPA predictors (i.e. risk perception, outcome expectancy and self-efficacy).

Furthermore, individuals who believe that an unhealthy behaviour can be compensated for by another healthy behaviour may not only be less motivated to change their behaviour (i.e. be less likely to form an intention), but may - also in spite of high intentions (Rabiau et al., 2006) - perceive a lower necessity to plan than individuals with lower CHBs. This is because in case of failure to change the behaviour due to lower planning, this failure might also be believed to be compensated for by other healthy behaviours. This is in line with Gollwitzer and Sheeran (2006) who assume that variables such as anticipated regret, self-evaluation or monitoring might influence whether planning strategies are formed or not.

Moreover, the CHB model (Rabiau et al., 2006) also states that the use of CHBs in the long-run is assumed to reduce a certain healthy behaviour, such as regular physical activity and in turn enhance negative health behaviours, such as sedentary behaviours. This is due to the assumption that individuals usually fail to engage in the intended compensatory behaviour (e.g. go to the gym the next day) because of procrastination or because the initially felt dissonance weakens over time (Rabiau et al., 2006). According to this, some of the studies investigating the effects of CHBs also investigated this direct association between CHBs and behaviour (Knäuper et al., 2004; Rabiau et al., 2009). All of them showed that CHBs were significantly associated with the behaviour even in competition with other predictors, like selfefficacy (Tăut \& Băban, 2008). For example, results by Knäuper et al. (2004) showed that the subscale 'substance use' of the CHBs which includes items on smoking and alcohol consumption, was significantly positively related to smoking-/alcohol-related risk behaviour. In addition, findings by Rabiau et al. (2009) also confirm a negative association of diabetic-specific CHBs with glucose testing frequency and metabolic control in adolescents with type 1 diabetes. On the basis of these theoretical specifications and empirical evidence, we assumed that CHBs are also directly associated with the behaviour. 


\section{Hypothesised model}

Based on the theoretical assumptions within the CHB model and the current state of research, it is assumed that $\mathrm{CHBs}$ are an important factor for intention formation. Since, however, the theory and the empirical findings additionally suggest a possible influence in the volition phase and on behaviour, this relationship will be analysed as well. Thus, the following model was defined to examine the association of the CHBs with other constructs: smoking-specific CHBs, risk perception, positive outcome expectancy, and self-efficacy were specified as predictors of the intention to stop smoking. The intention was specified as a predictor of action planning, whereas action planning was specified as a predictor of smoking behaviour. In addition, within the model it was tested whether smoking-specific CHBs are also related to planning and smoking behaviour. Furthermore, the model specified the indirect effect of the smoking-specific CHBs on planning via intention and on smoking behaviour, as well as the indirect effect of intention on smoking behaviour via planning (cf. Figure 1).

\section{Methods}

\section{Design and participants}

For data collection, the largest CATI-recruited (computer-assisted telephone interview) Internet panel in Switzerland was used. Sampling, data collection and data preparation were performed by the LINK Institute, an independent social research agency. Participants were invited by email. Younger adolescents were additionally recruited by means of flyers (e.g. in occupational career offices) and advertisements on smoking-related Swiss websites for youth. All participants could select compensation worth $30 \mathrm{CHF}$ ( $\$ 28 \mathrm{USD}$; such as a voucher for mobile phone credit or for a supermarket) if they participated in both points of measurement. At the first point of measurement (Time 1, T1), adolescents were only included if they were current smokers and had smoked at least 100 cigarettes in their life, which is a useful indicator of established smoking (Bondy, Victor, \& Diemert, 2009). After 4 months, participants were invited by email to the second point of measurement (Time 2, T2). Participation in the study involved providing voluntarily anonymous and confidential responses to questionnaires. All participants were treated according to the ethical guidelines of the American Psychological Association (APA, 2002).

A total of $N=480$ adolescents aged 15-20 years participated at Time 1, and 285 $(59.4 \%)$ of those also participated at Time 2. To test whether the longitudinal subsample was comparable to the initial sample, we compared the dropouts to the continuers. The dropouts did not differ significantly in relation to either sociodemographic factors or to any of the study-relevant variables, such as intention, number of smoked cigarettes or CHBs at the time of measurement T1 (all $p>0.50$ ). For our analysis, cases with inconsistent data $(n=33)$ were excluded (e.g. if an adolescent reported his/her age to be 18 at Time 1 and 22 at Time 2, or if the declared number of smoked cigarettes was not considered truthful, because it was too high for a daily/weekly consumption). In addition, for the analyses we excluded participants who smoked less than one cigarette per week at both points of measurement $(n=62)$. Therefore, the longitudinal subsample for this study consisted of 385 participants (191 male, 194 female) with a mean age of 17.80 years $(\mathrm{SD}=1.65)$. Most of the 
adolescents were Swiss (90.4\%). Approximately, half of the sample was currently in an apprenticeship (54.5\%) and $11.8 \%$ were pupils. The majority declared placing a high value on their own health (4-point Likert scale ranging from 1 to $4 ; M=3.42$; $\mathrm{SD}=0.67)$. The mean number of smoked cigarettes per day for all adolescents participating was $6.72(\mathrm{SD}=6.18 ; M d=5.0)$ at Time 1 , and $6.57(\mathrm{SD}=6.45$; $M d=5.0)$ at Time 2 . Two hundred and ten $(54.5 \%)$ adolescents were daily smokers and $175(45.5 \%)$ were occasional smokers.

\section{Measures}

CHBs, conditional risk perception, positive outcome expectancies, preaction selfefficacy and intention were assessed at Time 1 because they reflect the motivational phase of the HAPA. The other measures were assessed at Time 2. Unless otherwise noted, for most scales, the response format was a 4-point Likert scale ranging from 1 (disagree) to 4 (agree). In Table 1, the means, standard deviations, and alpha coefficients for all scales are displayed.

\section{Smoking-specific CHB scale}

The scale consists of 10 items (cf. Table A.1 in Appendix). An example item is 'The negative effects of smoking can be made up for by eating healthy' (Radtke et al., 2011). The response format was a 5-point Likert scale ranging from 1 (disagree) to 5 (agree). Of the 10 items, 2 items ('The negative effects of smoking can be reduced by exercising' and 'It is alright to smoke when going out as long as one smokes less or not at all the next day') were excluded in this study, because of low factor loadings $(<0.40)$. The analysis of internal consistency, as well as the modification indices in Mplus (L.K. Muthén \& B.O. Muthén, 2007) also suggested excluding both items. The content of the two items was still covered by the remaining items, which made it feasible to exclude both items.

\section{Risk perception}

Conditional risk perception was assessed with three items. An item example is the following: 'If I keep up smoking the way it is, there is a high likelihood that I will develop severe health problems' (Scholz et al., 2009).

\section{Positive outcome expectancies}

Four items were used to measure the positive outcome expectancies regarding quitting smoking. All items had the preface 'If I quit smoking...' followed by positive consequences such as '... I will simply feel better physically' (Copeland, Brandon, \& Quinn, 1995).

\section{Smoking-specific self-efficacy}

Three different kinds of smoking-specific self-efficacy were chosen to measure phasespecific smoking-specific self-efficacy (Scholz, Sniehotta, \& Schwarzer, 2005). First, the item stem 'I could manage to refrain from smoking...' was followed by items such as 'even if I have sorrows' for measuring preaction self-efficacy (four items). 
Table 1. Scale characteristics (mean, SD, range, Cronbach's alpha, $\alpha$ ).

\begin{tabular}{llccr}
\hline Scale & $M$ & SD & Range & $\alpha$ \\
\hline Smoking specific CHBs (T1) & 2.10 & 0.84 & 15 & 0.89 \\
HAPA variables & 2.32 & 0.89 & 14 & 0.93 \\
Conditional risk perception (T1) & 2.62 & 0.83 & 14 & 0.85 \\
Positive outcome expectancies (T1) & & & & \\
Smoking specific self efficacy & 2.45 & 0.85 & 14 & 0.87 \\
$\quad$ Preaction (T1) & 2.63 & 0.88 & 14 & 0.81 \\
$\quad$ Maintenance (T2) & 2.63 & 0.86 & 14 & 0.72 \\
$\quad$ Recovery (T2) & 1.78 & 0.93 & 14 & 0.87 \\
Intention (T1) & 2.31 & 0.98 & 14 & 0.84 \\
Planning (T2) & 6.57 & 6.45 & 130 & \\
Cigarettes per day (T2) & & & & \\
\end{tabular}

Note: CHBs, Compensatory Health Beliefs; T1 or T2, Time 1 or Time 2 of measurement.

Second, participants were required to rate their feelings of competency regarding the maintenance of not smoking (two items) with the item stem 'I am confident that I am able to ...' followed by items such as '... quit smoking even if I would have to put much efforts to overcome my current habits'. Third, recovery self-efficacy (two items) was assessed with the item stem 'I am confident that I am able to...' followed by items like '... return to quit smoking even if I have smoked once in a while' (Schwarzer, 2004).

\section{Intention}

Two items assessed the intention to quit smoking: the items are 'I intend to quit smoking immediately' and 'I intend to stop smoking completely' (Scholz et al., 2009).

\section{Action planning}

Planning was measured by four items. The item stem 'I have already made concrete plans...' was followed by items regarding 'when, where, and how not to smoke' as well as regarding 'when I will quit smoking' (Scholz et al., 2009).

\section{Self-reported smoking behaviour}

The questions were extracted from 'Tobacco Monitoring Switzerland [TMS].' Participants were asked, 'On average, how many cigarettes do you smoke per day?' Basic demographic information was also collected using the TMS questions (Tobacco Monitoring Switzerland, 2008).

\section{Data analysis}

To test the hypothesised model, structural equation modelling (SEM) with latent variables was employed with Mplus 6.01 (L.K. Muthén \& B.O. Muthén, 2007). All latent variables in the model were specified with the described variables mentioned below and were freely estimated. For evaluation of the model-fit, goodness-of-fit 
indices are presented, such as the $\chi^{2}$-test, the comparative fit index (CFI), the Tucker Lewis index (TLI), the root mean square error of approximation (RMSEA) and the standardised root mean square residual (SRMR). Since the $\chi^{2}$-test is an index dependent on sample size, it cannot be used exclusively as the criterion for determining model fit (Kline, 2005). Bollen and Long (1993) recommend that $\chi^{2}$ should not be larger than two to five times the degrees of freedom. According to $\mathrm{Hu}$ and Bentler (1999), a satisfactory model fit is indicated by a CFI and TLI $>0.90$, a RMSEA $<0.08$, and a SRMR $<0.05$. Hypothesised mediations were tested together because using multiple mediations is the most appropriate analytic strategy (Preacher \& Hayes, 2008). Also, bootstrapping was used to test the strength and significance of the indirect effects because it provides the most powerful method of obtaining confidence limits for indirect effects (Preacher \& Hayes, 2008). The lower and upper levels of the $95 \%$ confidence interval were reported.

Complete deletion of those participants holding missing values is only feasible when the assumption of missing completely at random (MCAR) is fulfilled (Little \& Rubin, 2002). This assumption, however, is only rarely met and as it cannot be tested directly, assuming a missing pattern of missing at random seems to be more appropriate (Graham, 2009). Thus, to account for missing values, the Full Information Maximum Likelihood technique (FIML), which is an integral part of Mplus, was applied. The FIML estimates model parameters on the basis of all available information of all observed (complete and incomplete) cases. As no systematic missing mechanisms could be detected in our dropout-analyses, the inclusion of auxiliary variables that might account for these potential missing patterns was not necessary (Graham, 2009).

\section{Results}

\section{Inter-correlations of latent constructs}

Table 2 presents the inter-correlations of the latent variables. Intention was significantly correlated with smoking-specific CHBs, positive outcome expectancies, and phase-specific self-efficacy beliefs but not with conditional risk perception. As shown, adolescent smokers holding higher smoking-specific CHBs have lower intentions to stop smoking. Furthermore, the results indicated that the lower the positive outcome expectancies, and the phase-specific self-efficacy beliefs, the higher the smoking-specific CHBs. On the other hand, a lower conditional risk perception was associated with a higher positive outcome expectancy and smoking-specific preaction self-efficacy. Moreover, smoking-specific CHBs were negatively correlated with planning, but not with the number of smoked cigarettes per day.

\section{Hypothesised model integrating smoking-specific CHBs into the HAPA}

The tested model integrating smoking-specific CHBs into the HAPA, fit the data well $\left(\chi^{2}(387, N=385)=596.99 ; p<0.00 ; \mathrm{CFI}=0.96\right.$, TLI $=0.96$, RMSEA $=0.04$, SRMR $=0.07$ ). All values met the criteria for acceptable model fit (Bollen \& Long, 1993; Hu \& Bentler, 1999). Figure 1 presents the parameter estimates (standardised solution).

In terms of correlations, smoking-specific CHBs were significantly negatively correlated with other predictors of intention: smoking-specific preaction self-efficacy 


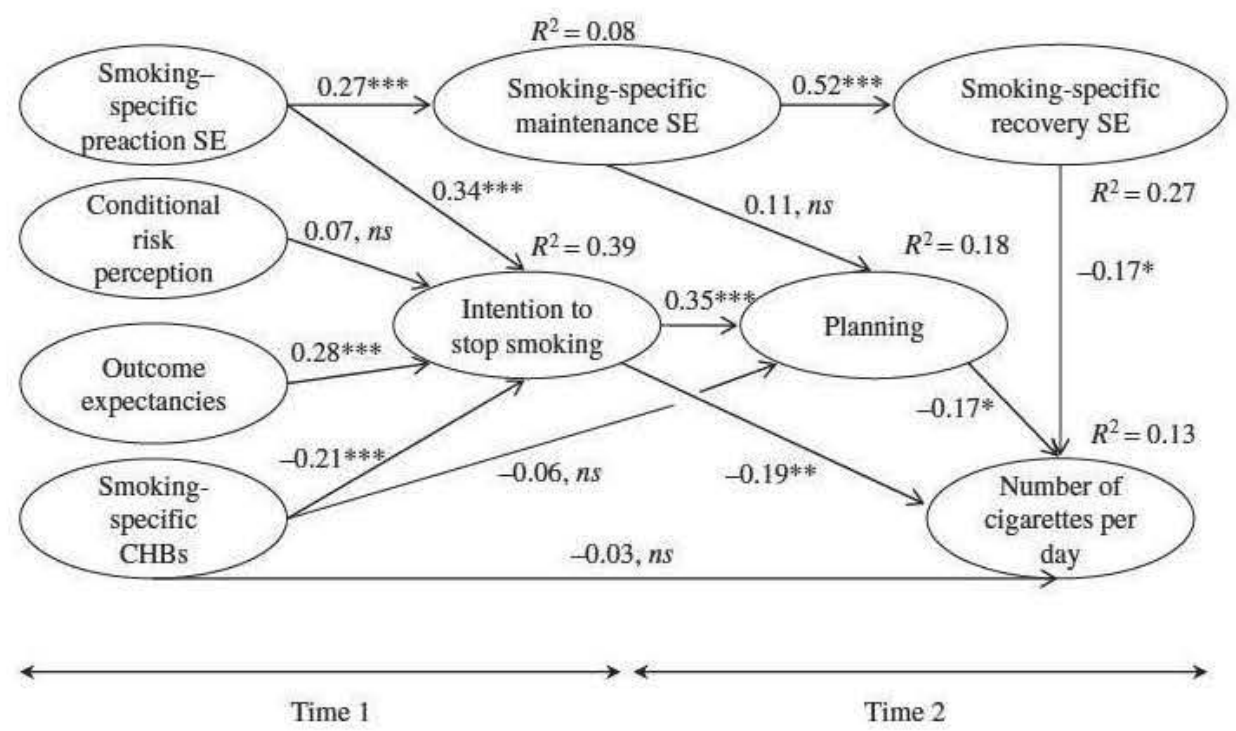

Figure 1. Hypothesised model with smoking specific CHBs for smoking in adolescents. SE, self efficacy; CHBs, Compensatory Health Beliefs. Standardised solution. The correlations between the predictors of intention are not displayed. $N 5000$ Bootstrapping resamples. ${ }^{*} p<0.05 ;{ }^{* *} p<0.01 ;{ }^{* * *} p<0.001$.

$(r=-0.23, p<0.01)$, and positive outcome expectancies $(r=-0.23, p<0.01)$, but not with conditional risk perception $(r=-0.01, p=0.91)$. Smoking-specific preaction self-efficacy was also significantly, positively associated with positive outcome expectancies $(r=0.46, p<0.01)$, and negatively with conditional risk perception $(r=-0.15, p<0.01)$. Moreover, positive outcome expectancies were significantly negatively correlated with conditional risk perception $(r=-0.23$, $p<0.01)$. The three self-efficacy beliefs were also interrelated: smoking-specific preaction self-efficacy accounted for $8 \%$ of the variance of smoking-specific maintenance self-efficacy, which in turn explained $27 \%$ of the variance of smoking-specific recovery self-efficacy.

Intention was predicted by smoking-specific preaction self-efficacy, positive outcome expectancies, and smoking-specific CHBs, accounting for $39 \%$ of the variance altogether. Conditional risk perception was not related to the intention to stop smoking. However, smoking-specific $\mathrm{CHBs}$ were negatively associated with intention $(\beta=-0.21, p<0.01)$ over and above the HAPA specific predictors, indicating that adolescent smokers holding higher smoking-specific $\mathrm{CHBs}$ have lower intentions to stop smoking. In contrast to our hypothesis, however, smokingspecific CHBs were not associated with planning $(\beta=-0.06, n s)$. Overall, $17 \%$ of the variance of planning to stop smoking was explained by intention. Furthermore, $13 \%$ of the variance in the number of smoked cigarettes per day was explained by planning, smoking-specific recovery self-efficacy, intention and smoking-specific CHBs, although smoking-specific CHBs were not directly significantly associated with smoking behaviour $(\beta=-0.03, n s)$.

As mentioned above, the indirect effects of smoking-specific CHBs on planning via intention and on smoking behaviour via intention and planning as well as the indirect effects of intention on smoking behaviour via planning were tested. 
Table 2. Intercorrelations between latent variables used in the SEM.

\begin{tabular}{|c|c|c|c|c|c|c|c|c|}
\hline & 1 & 2 & 3 & 4 & 5 & 6 & 7 & 8 \\
\hline 1. S spec. CHBs (T1) & 1.00 & & & & & & & \\
\hline 2. C. risk perception (T1) & 0.01 & 1.00 & & & & & & \\
\hline 3. Pos. outcome ex. (T1) & $0.23 * *$ & $0.23 * *$ & 1.00 & & & & & \\
\hline 4. Preaction $\mathrm{SE}(\mathrm{T} 1)$ & $0.23 * *$ & $0.15^{* *}$ & $0.46 * *$ & 1.00 & & & & \\
\hline 5. Maintenance SE (T2) & 0.12 & 0.07 & $0.16^{*}$ & $0.19 * *$ & 1.00 & & & \\
\hline 6. Recovery SE (T2) & 0.10 & $0.16^{*}$ & $0.30^{* *}$ & $0.32 * *$ & $0.38 * *$ & 1.00 & & \\
\hline 7. Intention (T1) & $0.34 * *$ & 0.04 & $0.42 * *$ & $0.45^{* *}$ & $0.16^{*}$ & $0.29 * *$ & 1.00 & \\
\hline 8. Planning (T2) & $0.16^{*}$ & 0.07 & $0.22 * *$ & $0.26^{* *}$ & 0.13 & $0.18^{* *}$ & $0.33 * *$ & 1.00 \\
\hline 9. Cigarettes per day (T2) & 0.10 & $0.34 * *$ & $0.41 * *$ & $0.29 * *$ & $0.17 *$ & $0.23 * *$ & $0.26 * *$ & $0.25 * *$ \\
\hline
\end{tabular}

Notes: S spec. CHBs, smoking specific Compensatory Health Beliefs; C., conditional; Pos. outcome ex., positive outcome expectancies; SE, smoking specific self efficacy. T1 or T2, Time 1 or Time 2 of measurement.

$* p<0.05 . * * p<0.01$ 
Results from bootstrapping provided evidence that smoking-specific CHBs were mediated via intention on planning $(\beta=-0.07, p<0.01,95 \%$ CI from -0.12 to $-0.03)$. In contrast, smoking-specific CHBs were not mediated on smoking behaviour via intention $(\beta=0.04, p=0.03,95 \% \mathrm{CI}$ from 0.00 to 0.08$)$ or planning ( $\beta=0.01, p=0.53,95 \%$ CI from -0.02 to 0.04 ), nor on smoking behaviour via intention and planning ( $\beta=0.01, p=0.08,95 \% \mathrm{CI}$ from -0.00 to 0.03 ). Moreover, planning did not mediate the association between intention and behaviour ( $\beta=-0.06, p=0.05,95 \%$ CI from -0.12 to 0.00$)$, but intention had a significant direct effect on behaviour $(\beta=-0.19, p<0.01)$.

\section{Discussion}

The first aim of this study was to investigate the effect of smoking-specific CHBs compared to other variables of a well-established behaviour change model like the Health Action Process Approach. To the best of our knowledge, this study is the first to examine such an effect. The results of the hypothesised model demonstrated that smoking-specific CHBs were significantly, negatively related to the intention to quit smoking over and above standard predictors of the HAPA. This confirms previous findings stating that smoking-specific $\mathrm{CHBs}$ are associated with readiness to stop smoking (Radtke et al., 2011). As expected, smoking-specific CHBs also showed a significantly negative association with smoking-specific preaction self-efficacy and positive outcome expectancies (Knäuper et al., 2004; Radtke et al., 2011). All three variables accounted for $39 \%$ of the variance in intention to quit smoking. Moreover, $13 \%$ of the variance in smoking behaviour was explained by planning and smokingspecific recovery self-efficacy. Furthermore, bootstrapping provided evidence that intention mediated the negative relation between smoking-specific $\mathrm{CHBs}$ and planning. In contrast, no significant direct or indirect effect of CHBs on the number of smoked cigarettes was found.

The smoking-specific CHB scale is proven to have good predictive validity (Radtke et al., 2011). In this study, however, two items of the scale had low factor loadings and the analysis of internal consistency suggested excluding both items. Nevertheless, the data provide further evidence that the scale of smoking-specific CHBs is a valid measure because the different compensatory strategies were still covered by all of the remaining items. It is supposed that only the item wording of the two excluded items might need revision. Therefore, the use of the shortened smoking-specific CHB scale in the structural equation model is maintainable, but we also recommend further investigation of the smoking-specific $\mathrm{CHB}$ scale in other samples.

Comparable to other studies (Schwarzer \& Luszczynska, 2008) the association between conditional risk perception and the intention to stop smoking was not significant. As Schwarzer (1992) mentioned, risk perception is the distal starting point of a deliberation process for health behaviour change and thus is not sufficient to generate an intention. Another explanation might be the item phrasing. Because of the young age of the participants, it is possible that the health consequences (heart attack, serious health damage) used in the item wording had no relevance for this age group, since these are long-term health consequences. In future research, the item wording should be adapted to adolescents (e.g. 'If I keep smoking the way I do, there is a high likelihood that I will get stained teeth'). Furthermore, the results of the 
correlations indicated that conditional risk perception was significantly negatively correlated with the other predictors of intention, with the exception of smoking-specific CHBs. This might be explained by the resulting significant positive correlation between conditional risk perception and the number of cigarettes smoked per day (cf. Table 2). It seems that smokers who smoked more cigarettes per day had a higher conditional risk perception, which might be caused by realistic estimations about the threat to their health. In addition, those who smoked a higher number of cigarettes per day reported lower levels of positive outcome expectancies and smoking-specific self-efficacy. For this reason, it can be concluded that smokers have a realistic risk perception concerning their smoking behaviour but that this is not sufficient for the formation of the intention to stop smoking.

An unexpected result was the non-significance of the mediation of intention on smoking behaviour via planning. This might be due to the fact that this study's sample was not a highly intentional sample. According to the HAPA, the addition of self-regulation strategies, like planning or action control, mainly results in notable effects if these strategies correctly address individuals who are in the volitional stage of the HAPA (Scholz, Schüz, Ziegelmann, Lippke, \& Schwarzer, 2008; Schwarzer \& Luszczynska, 2008). As Sheeran, Webb, and Gollwitzer (2005) pointed out, planning only plays a significant role in people with a high intention to quit.

In sum, the presented findings confirm the assumptions of the HAPA model regarding smoking behaviour in adolescents (Scholz et al., 2009; Schwarzer \& Luszczynska, 2008). Moreover, the central result of the SEM demonstrated that smoking-specific CHBs were a significant negative predictor of the intention to stop smoking over and above standard HAPA variables. Thus, upon replication, CHBs might be considered in future studies.

\section{Limitations}

Potential limitations of this study should be considered. First, a problem when analysing data based on self-reports is the assessment of the number of smoked cigarettes and the smoking status. In general, self-reports of adolescents' smoking behaviour are rather accurate in most studies (Bauman, Koch, \& Bryan, 1982; Post et al., 2005). Likewise, online surveys are useful tools for reaching young smokers and for estimating smoking behaviour. One advantage of online surveys is their relative anonymity (Klein, Thomas, \& Sutter, 2007). Nevertheless, the estimation of the number of cigarettes smoked relies on only one question. In SEM, the specification of the latent variable using only one manifest variable supposes a perfect measurement. In order to lower this measurement limitation, the question concerning the number of cigarettes smoked per day was complemented by a second question concerning the number of cigarettes smoked per week. This question was placed on another page of the online questionnaire to reduce memory effects. However, biochemical assessment should be considered in future studies (e.g. saliva cotinine; Patrick et al., 1994) to improve accuracy.

As a second limitation, the expandable sample size of adolescent smokers should be taken into account. For a longitudinal study with smokers between ages 15 and 
21, the sample size is acceptable because it is comparable to other studies (Schwarzer \& Luszczynska, 2008).

Third, this study is based on longitudinal data, but we did not analyse the behavioural changes of smokers. Changes should be analysed for studies interested in the effects of interventions. Because this study is based on non-intervention data, the baseline behaviour of smoking, which was already measured, was not included in the analysis. This was done because its inclusion in the analyses could have masked the effects of the other predictor variables (Bandura, 1997). Furthermore, instead of measuring all social-cognitive predictors at all points of measurement, the motivational variables were assessed at Time 1 only, whereas the volitional variables were assessed at Time 2 only. This was also done due to the correlational nature of the study. It was not the aim of this study to measure the change of social-cognitive predictors, but to examine whether smoking-specific CHBs were able to add to the explained variance in intention and smoking behaviour over and above HAPA-specific predictors. Future studies might want to include all variables at all points of measurement in order to examine whether change in HAPA variables and change in $\mathrm{CHBs}$ is associated with change in intentions and in behaviour (Scholz et al., 2008).

Although it is quite common to use only two measurements for longitudinally testing a model, one should be aware of potential limitations (e.g. identification and estimation problems) that can emerge when using only two measurements (Ciesla, Cole, \& Steiger, 2007). Finally, due to the correlational design of the study, no causal inferences can be drawn.

\section{Outlook and conclusions}

This study presents primary evidence of the usefulness of smoking-specific CHBs in adolescents compared to other well-established constructs embedded in the HAPA model. As a consequence, there are implications for future research. Because the present results provide only a first confirmation of the relevance of $\mathrm{CHBs}$, further research is recommended to examine smoking-specific CHBs in different samples (e.g. in adults).

Many smoking intervention programmes for adolescents are aimed at increasing self-efficacy, exploring the pros and cons of smoking and quitting, or enhancing knowledge about smoking (Colby et al., 2005; Dijkstra, De Vries, \& Roijackers, 1998; Patten et al., 2008). The results presented in this article also emphasise including the consideration of smoking-specific CHBs. CHBs should be unmasked in order to raise adolescents' awareness of CHBs' maladaptive effects. Avoiding CHBs should be stressed because first, there is no clear medical evidence that negative health effects of smoking can truly be compensated for by another behaviour and second, people often do not manage to engage in the compensating healthy behaviour. Alternative strategies should be implemented in order to keep smokers from automatically activating CHBs if they fail to resist the temptations of smoking. Planning how to overcome temptation is a possible strategy for coping with situations in which it is difficult for smokers to resist the temptation of a cigarette (Gollwitzer \& Sheeran, 2006; Sniehotta et al., 2005) and might play a role in preventing the activation of CHBs (Tăut \& Băban, 2008). 


\section{Acknowledgements}

This study was supported by the 'Tabakpräventionsfonds' (Swiss Tobacco Prevention Fund; grant number 06.004657). For all authors, there is no conflict of interest, no financial or personal relation to people or organisations that could have influenced this study. The authors thank Angela Bearth and Désirée Füllemann for their assistance in collecting data.

\section{References}

American Cancer Society (2000). Cancer facts and figures. New York, NY: American Cancer Society.

American Psychological Association (2002). Ethical principles of psychologists and code of conduct. American Psychologist, 57, 10601073.

Bak, J., \& Piko, B. (2007). Smoke free world for children's welfare: Perceptions of smoking in preadolescence. Children and Youth Services Review, 29, 283293.

Bandura, A. (1997). Self efficacy: The exercise of control. New York, NY: Freeman.

Bauman, K.E., Koch, G.G., \& Bryan, E.S. (1982). Validity of self reports of adolescent cigarette smoking. Substance Use and Misuse, 17, 11311136.

Bollen, K., \& Long, J. (Eds.). (1993). Testing structural equation models. Thousand Oaks, CA: Sage.

Bondy, S.J., Victor, J.C., \& Diemert, L.M. (2009). Origin and use of the 100 cigarette criterion in tobacco surveys. Tobacco Control, 18, 317323.

Ciesla, J.A., Cole, D.A., \& Steiger, J.H. (2007). Extending the trait state occasion model: How important is within wave measurement equivalence? Structural Equation Modeling, 14, 7797.

Colby, S.M., Montia, P.M., Tevyawa, T.O., Barnetta, N.P., Spiritoa, A., Rohsenowa, D.J., ..., Lewander, W. (2005). Brief motivational intervention for adolescent smokers in medical settings. Addictive Behaviors, 30, 865874.

Copeland, A.L., Brandon, T.H., \& Quinn, E.P. (1995). The smoking consequences questionnaire adult: Measurement of smoking outcome expectancies of experienced smokers. Psychological Assessment, 7, 484494.

Dijkstra, A., De Vries, H., \& Roijackers, J. (1998). Long term effectiveness of computer generated tailored feedback in smoking cessation. Health Education Research, 13, 207214.

Gollwitzer, P.M., \& Sheeran, P. (2006). Implementation intentions and goal achievement: A meta analysis of effects and processes. Advances in Experimental Social Psychology, 38, 249268.

Graham, J.W. (2009). Missing data analysis: Making it work in the real world. Annual Review of Psychology, 60, 549576.

Harmon Jones, E., \& Harmon Jones, C. (2007). Cognitive dissonance theory after 50 years of development. Zeitschrift für Sozialpsychologie, 38, 716.

Hu, L., \& Bentler, P.M. (1999). Cutoff criteria for fit indices in covariance structure analysis: Conventional criteria versus new alternatives. Structural Equation Modeling, 6(1), 155.

Keller, R., Radtke, T., Krebs, H., \& Hornung, R. (2011). Der Tabakkonsum der Schweizer Wohnbevölkerung in den Jahren 2001 bis 2010. Tabakmonitoring Schweizerische Umfrage zum Tabakkonsum [Tobacco consumption of the Swiss Population between 20012010. Tobacco Monitoring Switzerland]. Zurich, Switzerland: University of Zurich, Department of Psychology, Social and Health Psychology.

Klein, J.D., Thomas, R.K., \& Sutter, E.J. (2007). Self reported smoking in online surveys: Prevalence estimate validity and item format effects. Medical Care, 45, 691695.

Kline, R.B. (2005). Principles and practice of structural equation modeling. New York, NY: Guilford Press. 
Knäuper, B., Rabiau, M., Cohen, O., \& Patriciu, N. (2004). Compensatory health beliefs scale development and psychometric properties. Psychology and Health, 19, 607624.

Kronick, I., \& Knäuper, B. (2010). Temptations elicit compensatory intentions. Appetite, 54, 398401

Lee, W.B., Fong, G.T., Zanna, M.P., Borland, R., Omar, M., \& Sirirassamee, B. (2009). Regret and rationalization among smokers in Thailand and Malaysia: Findings from the International Tobacco Control Southeast Asia Survey. Health Psychology, 28, 457464.

Little, R.J.A., \& Rubin, D.B. (2002). Statistical analysis with missing data (2nd ed.). Hoboken, NJ: Wiley.

Mermelstein, R. (2003). Teen smoking cessation. Tobacco Control, 12, i25 i35.

Muthén, L.K., \& Muthén, B.O. (2007). Mplus. User's guide (5th ed.). Los Angeles, CA: Muthén \& Muthén.

Oakes, W., Chapman, S., Borland, R., Balmford, J., \& Trotter, L. (2004). Bulletproof sceptics in life's jungle: Which self exempting beliefs about smoking most predict lack of progression towards quitting? Preventive Medicine, 39, 776782.

Patrick, D.L., Cheadle, A., Thompson, D.C., Diehr, P., Koepsell, T., \& Kinne, S. (1994). The validity of self reported smoking: A review and meta analysis. American Journal of Public Health, 84, 10861093.

Patten, C.A., Decker, P.A., Dornelas, E.A., Barbagallo, J., Rock, E., Offord, K.P.,..., Pingree, S. (2008). Changes in readiness to quit and self efficacy among adolescents receiving a brief office intervention for smoking cessation. Psychology, Health and Medicine, 13, 326336.

Pinel, J.P.J., Assanand, S., \& Lehman, D.R. (2000). Hunger, eating, and ill health. American Psychologist, 55, 11051116.

Pitts, M., \& Phillips, K. (1998). The psychology of health. An introduction (2nd ed.). London: Routledge.

Post, A., Gilljam, H., Rosendahl, I., Meurling, L., Bremberg, S., \& Galanti, M.R. (2005). Validity of self reports in a cohort of Swedish adolescent smokers and smokeless tobacco (snus) users. Tobacco Control, 14, 114117.

Preacher, K.J., \& Hayes, A.F. (2008). Asymptotic and resampling strategies for assessing and comparing indirect effects in multiple mediator models. Behavior Research Methods, 40, 879891.

Rabiau, M., Knäuper, B., \& Miquelon, P. (2006). The external quest for optimal balance between maximizing pleasure and minimizing harm: The compensatory health beliefs model. British Journal of Health Psychology, 11, 139153.

Rabiau, M., Knäuper, B., Nguyen, T. K., Sufrategui, M., \& Polychronakos, C. (2009). Compensatory beliefs about glucose testing are associated with low adherence to treatment and poor metabolic control in adolescents with type 1 diabetes. Health Education Research, 24, 890896.

Radtke, T., Keller, R., Krebs, H., \& Hornung, R. (2010). Passivrauchen in der Schweizer Bevölkerung 2009. Tabakmonitoring Schweizerische Umfrage zum Tabakkonsum. [Second hand smoke in Switzerland. Tobacco Monitoring Switzerland]. Zurich, Switzerland: University of Zurich, Department of Psychology, Social and Health Psychology.

Radtke, T., Scholz, U., Keller, R., Knäuper, B., \& Hornung, R. (2011). Smoking specific compensatory health beliefs and the readiness to stop smoking in adolescents. British Journal of Health Psychology, 16, 610625.

Scheffel, J., \& Schou, K.C. (2007). To be one who continues to smoke: Construction of legitimacy and meaning in young adult's accounts of smoking. Addiction Research and Theory, 15, 161176.

Scholz, U., Nagy, G., Göhner, W., Luszczynska, A., \& Kliegel, M. (2009). Changes in selfregulatory cognitions as predictors of changes in smoking and nutrition behaviour. Psychology and Health, 24, 545561. 
Scholz, U., Schüz, B., Ziegelmann, J.P., Lippke, S., \& Schwarzer, R. (2008). Beyond behavioral intentions: Planning mediates between intentions and physical activity. British Journal of Health Psychology, 13, 479494.

Scholz, U., Sniehotta, F.F., \& Schwarzer, R. (2005). Predicting physical exercise in cardiac rehabilitation: The role of phase specific self efficacy beliefs. Journal of Sport and Exercise Psychology, 27, 135151.

Schwarzer, R. (1992). Self efficacy in the adoption and maintenance of health behaviors. Theoretical approaches and a new model. In R. Schwarzer (Ed.), Self efficacy: Thought control of action (pp. 217 242). Washington, DC: Hemisphere.

Schwarzer, R. (2004). Psychologie des Gesundheitsverhaltens. Einführung in die Gesundheitspsychologie [Introduction into health psychology] (3rd ed., revised). Göttingen, Germany: Hogrefe.

Schwarzer, R. (2008). Modelling health behaviour change: How to predict and modify the adoption and maintenance of health behaviour. Applied Psychology: An International Review, 57(1), 129.

Schwarzer, R., \& Luszczynska, A. (2008). How to overcome health compromising behaviours. The health action process approach. European Psychologist, 13, 141151.

Schwarzer, R., Schüz, B., Ziegelmann, J.P., Lippke, S., Luszczynska, A., \& Scholz, U. (2007) Adoption and maintenance of four health behaviors: Theory guided longitudinal studies on dental flossing, seat belt use, dietary behavior, and physical activity. Annals of Behavioral Medicine, 33, 156166.

Shafey, O., Eriksen, M., Ross, H., \& Mackay, J. (2009). The tobacco atlas (3rd ed.). Atlanta, GA: American Cancer Society.

Sheeran, P., Webb, T.L., \& Gollwitzer, P.M. (2005). The interplay between goal intentions and implementation intentions. Personality and Social Psychology Bulletin, 31, 8798.

Sniehotta, F.F., Schwarzer, R., Scholz, U., \& Schüz, B. (2005). Action planning and coping planning for long term lifestyle change: Theory and assessment. European Journal of Social Psychology, 35, 565576.

Tabachnik, B.G., \& Fidell, L.S. (2007). Using multivariate statistics (5th ed.). Boston, MA: Pearson.

Tăut, D., \& Băban, A. (2008). Examination of the relationship between self regulation strategies and healthy eating patterns in coronary heart disease patients. The relevance of compensatory health beliefs. Cognition, Brain and Behavior, 12, 219231.

Tobacco Monitoring Switzerland. (2008). Catalogue of questions in the basic module de. Zurich, Switzerland: University of Zurich, Department of Psychology. Retrieved from http://www.tabakmonitoring.ch/Fragenkatalog/Fragenkatalog_Basismodul_de.pdf

WHO (2007). The European tobacco control report. Copenhagen, Denmark: WHO Regional Office for Europe. 
Appendix

Table A.1. The smoking specific CHB scale.

\begin{tabular}{ll}
\hline Subscales & \multicolumn{1}{c}{ Items } \\
\hline Exercise & $\begin{array}{l}\text { 1. Smoking can be compensated for by physical activity. } \\
\text { 2. The negative effects of smoking can be reduced by exercising. } \\
\text { 3. The negative effects of smoking can be balanced by exercising } \\
\text { vigorously and regularly every week. }\end{array}$ \\
Food and drink & $\begin{array}{l}\text { 4. Smoking is ok if one drinks less alcohol in exchange. } \\
\text { 5. It is alright to smoke if one takes in enough vitamins. } \\
\text { 6. The negative effects of smoking can be made up for by }\end{array}$ \\
eating healthy. & $\begin{array}{l}\text { 7. Smoking from time to time is ok if one eats healthy. } \\
\text { 8. The effects of smoking during the weekend can be compensated } \\
\text { for by not smoking during the week. }\end{array}$ \\
9. It is alright to smoke when going out as long as one smokes \\
less or not at all the next day.
\end{tabular}

Notes: The items 2 and 9 were excluded in this study, because of low factor loadings $(<0.40)$. This scale was originally published in the British Journal of Health Psychology (Radtke et al., 2011). Permission to reprint was granted by the publisher. 